\title{
1 Powerful eQTL mapping through low coverage RNA sequencing
}

2
Tommer Schwarz ${ }^{1,2}$, Toni Boltz ${ }^{3}$, Kangcheng Hou ${ }^{1}$, Merel Bot ${ }^{4}$, Chenda Duan ${ }^{5}$, Loes Olde Loohuis $^{7,8}$, Marco P. Boks ${ }^{9}$, René S. Kahn ${ }^{9,10}$, Roel A. Ophoff ${ }^{1,3,11}$, Bogdan Pasaniuc ${ }^{1,2,3,6}$

1. Bioinformatics Interdepartmental Program, University of California Los Angeles, Los Angeles, CA, USA

2. Department of Computational Medicine, David Geffen School of Medicine, University of California Los Angeles, Los Angeles, CA, USA

3. Department of Human Genetics, David Geffen School of Medicine, University of California Los Angeles, Los Angeles, CA, USA

4. Center for Neurobehavioral Genetics, Semel Institute for Neuroscience and Human Behavior, University of California, Los Angeles, California, USA

5. Department of Computer Science, University of California, Los Angeles, Los Angeles, CA USA 6. Department of Pathology and Laboratory Medicine, David Geffen School of Medicine, University of California Los Angeles, Los Angeles, CA, USA

7. Department of Psychiatry, David Geffen School of Medicine, University of California, Los Angeles, Los Angeles, CA, USA

8. Program in Neurobehavioral Genetics, Semel Institute, David Geffen School of Medicine, University of California, Los Angeles, Los Angeles, CA, USA

9. Department of Psychiatry, Brain Center University Medical Center Utrecht, University Utrecht, Utrecht, The Netherlands

10. Department of Psychiatry, Icahn School of Medicine, Mount Sinai, NY, the United States of America

11. Department of Psychiatry, Erasmus University Medical Center, Rotterdam, The Netherlands

Corresponding authors: Tommer Schwarz (tomschwarz@g.ucla.edu), Bogdan Pasaniuc (pasaniuc@ucla.edu) 


\section{ABSTRACT}

Mapping genetic variants that regulate gene expression (eQTL mapping) in large-scale RNA

51 sequencing (RNA-seq) studies is often employed to understand functional consequences of

52 regulatory variants. However, the high cost of RNA-Seq limits sample size, sequencing depth,

53 and therefore, discovery power. In this work, we demonstrate that, given a fixed budget, eQTL

54 discovery power can be increased by lowering the sequencing depth per sample and increasing

55 the number of individuals sequenced in the assay. We perform RNA-Seq of whole blood tissue

56 across 1490 individuals at low-coverage (5.9 million reads/sample) and show that the effective

57 power is higher than that of an RNA-Seq study of 570 individuals at high-coverage (13.9 million

58 reads/sample). Next, we leverage synthetic datasets derived from real RNA-Seq data to explore

59 the interplay of coverage and number individuals in eQTL studies, and show that a 10-fold

60 reduction in coverage leads to only a 2.5-fold reduction in statistical power. Our study suggests

61 that lowering coverage while increasing the number of individuals is an effective approach to

62 increase discovery power in RNA-Seq studies.

\section{KEYWORDS}

64 RNA-Seq, Gene expression, Association testing

\section{BACKGROUND}

67 The vast majority of risk loci identified in genome-wide association studies (GWAS) are difficult to

68 interpret as they lie in noncoding regions of the genome. Variants that regulate gene expression

69 abundance, as measured through expression quantitative trait locus (eQTL) studies, provide

70 insightful information about the functional interpretation of GWAS signals ${ }^{1-2}$. By integrating eQTL

71 associations with GWAS, we can hope to identify target genes that are driving the GWAS signal

72 at a locus ${ }^{3-6}$. RNA sequencing (RNA-Seq) is the state-of-the-art assay for measuring gene 
73 expression in bulk tissue and is therefore the assay of choice for eQTL mapping ${ }^{7-8}$. However, the

74 high cost of RNA-Seq often limits the sample size and therefore reduces the discovery power of

75 eQTL studies based on RNA-Seq ${ }^{2,6,9}$.

76 Traditional RNA-Seq study design prioritizes sequencing depth per individual (targeted levels of

77 coverage in the range of 30-50 million reads) over the number of individuals (samples) included

78 in the study ${ }^{10-12}$. However, given that high levels of coverage per individual limits the sample size

79 of a study, this results in a loss of statistical power in eQTL mapping. Previous studies have

80 established that the low-coverage whole genome sequencing of a larger number of individuals

81 attains increased power of association compared to higher-coverage studies of smaller sample

82 sizes in GWAS ${ }^{13-17}$. This raises the hypothesis that, similarly as for whole genome sequencing

83 and GWAS, lower coverage RNA-seq with a considerable increase in the number of individuals

84 sequenced could increase power of discovery in eQTL studies ${ }^{18-21}$. Currently, there is no

85 systematic approach for determining the optimal sample size (in terms of number of sequenced individuals) and coverage to maximize eQTL discovery power.

87 In this work, we perform RNA-Seq in 1490 individuals at a lower coverage (average mapped read depth of 5.9 million reads/sample) and find that eQTL discovery power is better than that of an high-coverage RNA-Seq, we find a high degree of consistency in both the gene expression as

91 well as eQTL effects. We assess the interplay of coverage per sample and accuracy of expression

92 estimates using synthetic RNA-Seq datasets generated by the down-sampling of real high-

93 coverage data. Our analyses show that a sequencing experiment conducted with a target

94 coverage of 10 million reads/sample has an average correlation per-gene of 0.40 , when compared

95 to an experiment conducted with a target coverage of 50 million reads/sample. We provide

96 evidence to show that under a fixed budget, sequencing at lower coverage levels $(<10$ million 
97 reads/sample) and increased sample size can boost the effective sample size per unit of cost

98 compared to standard approaches of eQTL study design.

\section{RESULTS}

\section{Low-coverage RNA-sequencing is accurate for eQTL mapping}

101 To validate the utility of low-coverage RNA-sequencing, we sequenced whole blood tissue from

$102 \mathrm{~N}=1490$ unrelated individuals (Methods) (Supplementary Figure 1A and Supplementary

103 Figure 1B). We target a sequencing coverage of 9.5 million reads per sample, yielding $M=5.9$

104 million reads mapped to RefSeq genes on average (sd across samples of 1.96 million,

105 Supplementary Figure 2). We refer to this dataset as the lower-coverage RNA-Seq, or the M=5.9

106 million reads/sample dataset. We contrast this dataset with an RNA-Seq dataset obtained with a

107 similar budget, but with 2.4-fold higher coverage $(M=13.9$ reads $)$ across $N=570$ individuals

108 (Supplementary Figure 1C and Supplementary Figure 1D) ${ }^{22}$. We refer to this as the highercoverage whole blood RNA-Seq, or the $M=13.9$ million reads/sample dataset (Table 1 ).

110 First, we assess the number of genes quantified in the two datasets. We observe 40459 genes

111 with at least one mapped read on average across samples in the whole blood high-coverage

112 dataset, and 27308 genes with at least one mapped read on average across samples in the whole

113 blood low-coverage dataset. Notably, when restricting to protein coding genes with at least one

114 mapped read in both the high-coverage and low-coverage datasets, we find more similar numbers

115 between the data sets, with 18329 and 15605 genes quantified, respectively. This is likely due to

116 the very sparse abundance of the non-protein coding genes, making them less likely to be

117 detected in a lower coverage dataset. Indeed, we observe similar effects across the high vs low

118 coverage datasets when assessing the genes with sufficient expression to be included in eQTL

119 analysis (TPM > 0.1 in 20\% of individuals, see Methods): 26566 genes (15496 protein coding 120 genes) in high coverage data versus 19039 (13339 protein coding genes) in low coverage data. 
121 Most importantly we observe a high correlation in the abundance levels across the two data sets

122 thus demonstrating that high and low coverage recover similar expression $\left(R^{2}=0.91\right.$, Figure $\left.1 \mathrm{~A}\right)$.

123 Next, we investigate the power of low-coverage RNA-Seq for eQTL mapping. We conducted cis-

124 eQTL mapping with a $1 \mathrm{Mb}$ window using FastQTL, restricting to the 1490 unrelated individuals

125 in the low-coverage RNA-Seq data (Methods), to identify 7587 genes (eGenes) with a significant

126 association at FDR correction level of $5 \%$. As expected, eQTL distribution is concentrated at

127 transcription start sites (TSS), with $73 \%$ of eGenes TSS within $250 \mathrm{~kb}$ of the associated SNP

128 (eSNP). Repeating this approach using the high-coverage whole blood data in 570 individuals,

129 we only find 5971 genes with a significant association at FDR correction level of $5 \%$. 4969 of the

1307587 eGenes found using the low-coverage data are also significant in the high-coverage data.

131 Of these, 2151 of the eGenes are protein coding eGenes that share the same associated eSNP,

132 and we see an extremely high level of concordance between effect sizes for these eGenes across

133 the two datasets $\left(R^{2}=0.93\right.$, Figure $\left.1 B\right)$. This further indicates that low-coverage RNA-Seq is

134 robust in capturing eQTL effect sizes. 1002 genes were found to be eGenes in the high-coverage

135 eQTL analysis but not in the low-coverage analysis, with 573 (of the 1002) not passing expression

136 levels (TPM >0.1 in 20\% individuals) to be included in the low-coverage eQTL analysis; only 234

137 of the 573 were protein coding genes. Similar concordance is observed at the level of $p$-values

138 for the associations in both datasets (Figure 1C). Comparing the p-values for eGenes detected

139 in both eQTL analyses, the corresponding regression line has a slope of 0.39 , consistent with

140 lower-coverage dataset having superior statistical power to detect associations over lower-

141 coverage dataset, and consistent with overall number of significant eQTL discoveries. We report

142 the results from using typed SNPs in these eQTL analyses (Methods), but observe similar

143 patterns when using the full set of imputed SNPs.

144 To further validate the performance of eQTL analysis using lower coverage RNA-Seq (coverage

$1455.9 M, n=1490$ ), we compared the resulting eQTLs to the ones found by GTEx consortium in 
146 whole blood ${ }^{12}$ (Supplementary Figure 3). Restricting to the 12247 protein coding genes with

147 sufficient expression to be included in both studies (> 0.1 TPM in $20 \%$ of samples) we find that

1484529 out of the 5957 protein coding genes (76\%) with a significant association using the lower-

149 coverage data also had a significant association in GTEx, correcting at an FDR level of 5\%.

150 Further restricting to eGenes with the same leading SNP in both of these datasets (140 genes)

151 (Figure 1D), we observe a correlation $\left(R^{2}\right)$ of 0.85 between their effect sizes. Looking into the

1521428 protein coding genes with a significant association in eQTL analysis using the lower-

153 coverage RNA-Seq but not in GTEx using an FDR cutoff of 5\%, we observe that 372 have

154 significant association in GTEx using an FDR cutoff of $10 \%$. To further ensure that these eGenes

155 are not false positives, we compare the set of 1428 genes with eQTL analysis conducted by the

156 eQTLGen Consortium ${ }^{23}$ and find that all but 190 of these genes have been found to have a

157 significant association in eQTLGen. This suggests that the additional associations found using

158 lower-coverage data that are not found in GTEx are not false positives, but fall just below the

159 significance threshold in the GTEx analysis.

160 Finally, we explore the impact of RNA-Seq at lower coverages for cell type expression

161 estimation. We use Cibersort ${ }^{24}$ to compare cell-type proportion estimates between the lower-

162 coverage data and higher-coverage data (Methods). We find that the median estimated cell

163 type proportions are conserved across both datasets, suggesting that deconvolution of cell type

164 specific signal from gene expression profiles of whole blood samples is not impacted when

165 coverage is reduced by half (Supplementary Figure 7).

Impact of RNA-seq coverage on eQTL power

167 Having demonstrated the accuracy of low-coverage RNA-Seq in eQTL mapping in real data, we 168 next focused on exploring the interplay of number of individuals and coverage for optimizing 169 power for discovery. As simulating RNA-Seq data is challenging ${ }^{25-26}$, we down-sample reads from 170 higher-coverage RNA-Seq data to create synthetic datasets at various coverages (Methods). We 
171 observe that with just a fraction of the reads, it is still possible to estimate gene expression (Figure

172 2A). For example, using just $10 \%$ of the data (5.0 million reads/sample) retains a per gene $\mathrm{R}^{2}$ of

1730.40 , on average. The results from our analyses using these synthetic lower-coverage RNA-Seq

174 datasets suggest that under simplified settings of no per-sample library preparation cost, the

175 statistical power in an association study can be increased up to fourfold by spending more

176 resources on increasing sample size and fewer resources on increasing coverage. In practice,

177 increasing the number of samples in an RNA-Seq study leads to increased library preparation

178 costs, making the increase in obtainable statistical association power less obvious.

179 It has been established that statistical power in association studies is a function of sample size, 180 phenotype measurement accuracy, and genotype measurement accuracy ${ }^{13,19,29}$. This means that

181 the power of a study with sample size $\mathrm{N}$ and estimated gene expression is approximately the 182 same as the power of a study with sample size $\mathrm{N}$, using the true gene expression measurements 183 (Methods). In this scenario, $\mathrm{R}^{2}$ is the correlation between the true expression and the expression estimates. We therefore report the squared correlation $\left(R^{2}\right)$ between synthetic datasets at various coverages and the full data at an average of 50 million reads/sample (which is assumed to be the true gene expression). While these results show the mean $\mathrm{R}^{2}$ for all genes obtained under one synthetic dataset (one draw) per coverage level, we find that the synthetic datasets are consistent across multiple draws at the same coverage level (Supplementary Figure 4A) and each show similar correlations with the ground truth gene expression (Supplementary Figure 4B). explore the number of genes with significant associations after FDR correction at $5 \%$ under 192 various levels of simulated coverage (Figure 2B). Using synthetic data, as the number of reads 193 per sample decreases, we find that many eGenes are still detectable. For example, at 10 million 194 reads per sample, just $20 \%$ of the full coverage, $60 \%$ of the eGenes are still detected. In the 195 context of eQTL studies, synthetic RNA-Seq supports the idea that sequencing at lower 
coverages over a higher number of individuals is a promising approach to boosting statistical

197 power.

198 Finally, we explore the estimation accuracy in the synthetic data as a function of relative gene 199 expression abundance, since less abundant genes may not be captured altogether at lower 200 sequencing coverages. We stratify genes into five groups based on their relative expression in 201 the full dataset $\left(M=50.3\right.$ million reads/sample) and report the $R^{2}$ for genes in each of these groups 202 in synthetic data (Figure 2C). We observe that in the synthetic RNA-Seq dataset at 10 million

203 reads/sample, we capture expression of highly expressed genes better than lower expressed 204 genes. Specifically, for genes in the lowest through the highest quintiles of relative gene 205 abundance, we find the average correlation $\left(R^{2}\right)$ to the ground truth of expression to be $0.36,0.44$, $2060.61,0.73,0.86$, respectively. We observe the same effect for synthetic datasets at coverages of 2071 million reads/sample and 25 million reads/sample (Supplementary Figure $\mathbf{5 A}$ and 208 Supplementary Figure 5B). These results suggest that the ability to achieve similar power in 209 eQTL analysis studies will differ per gene, and is a function of relative expression. We further 210 investigate the properties of genes with quantification accuracy influenced by coverage levels of 211 sequencing and find that that protein coding genes are more accurately quantified at lower 212 coverage levels (Supplementary Figure 6A). Conversely, the number of transcripts per gene, 213 gene length, and GC content do not appear to be factors that broadly influence the gene 214 quantification accuracy when sequencing coverage is reduced (Supplementary Figure 6B, 215 Supplementary Figure 6C, and Supplementary Figure 6D).

216 Optimal association power for eQTLs is attained at lower coverage with a larger number 217 of samples

218 In the context of reducing experimental costs, we explored the trade-off between the number of 219 samples sequenced and the average coverage per sample. We evaluated the expected effective 220 sample size obtained with lower coverage per sample and compared this with a conventional 
221 approach of 50 million reads/sample. We down-sample reads (as described in Section 1 and

222 Methods) from a high-coverage RNA-Seq experiment derived from Fibroblast tissue in order to

223 create lower-coverage RNA-Seq synthetic data. This is done to match actual low coverage

224 sequencing as closely as possible. To evaluate the relationship between cost, coverage, and

225 sample size, we use the following equation to model the budget: $B=n * a+\frac{n * b * c}{d}$

226 Where $B$ is the cost/budget (in US dollars), $a$ is the library preparation cost per sample, $b$ is the

227 target coverage of each sample (in millions of reads), $c$ is the cost per lane (which contains $d$

228 million reads), and $d$ is the number of reads per sequencing lane (in millions). We compute the

229 effective sample size of an eQTL study as a function of average coverage, which determines the

230 number of samples sequenced under a fixed budget (Figure 3A). As an example, at a fixed

231 budget of $\$ 300,000$, the highest effective sample size is achieved by sequencing 2045 individuals

232 using 10 million reads per sample, which leads to a corresponding effective sample size of 1107.

233 An experiment achieving the sample effective sample size, using 50 million reads per sample,

234 would cost $\$ 426,564\left(\mathrm{~N}=1107, \mathrm{R}^{2}=1.0\right)$. Therefore, by lowering the coverage of each sample

235 and increasing sample size, we achieve the same effective sample size at just $70.3 \%$ of the cost.

236 In practice, it is common to observe a considerable discrepancy between the target number of

237 reads in an experiment and the number of reads that successfully map to genes. This can be

238 attributed to different library prep techniques, quality of samples, or tissue type. To show how

239 mapping rate can influence the effective sample size of an experiment, we model effective sample

240 size with varying levels of mapping rates (Methods). As expected, we observe that as the

241 mapping rate increases, there is a corresponding increase in effective sample size (Figure 3C).

242 We provide a webtool as a practical approach for selecting cost-effective designs for maximizing

243 eQTL power: https://tomschwarz.shinyapps.io/RNASeqCoverageCalculator/. 
244 With a budget of $\sim \$ 300 \mathrm{k}$ and an expected mapping rate of 0.60 (chosen based on mapping rate

245 of similar experiments using TruSeq Stranded plus rRNA and GlobinZero in whole blood tissue),

246 we see the maximum effective sample size would be achieved at a target coverage of 7 million

247 reads per sample, including 2227 individuals in the study. We estimate that achieving the same

248 effective sample size using data with 50 million reads per sample would cost $\sim \$ 500 \mathrm{k}(\mathrm{N}=1328)$,

249 or $1.78 x$ the cost of sequencing 2227 individuals at a coverage of 7 million reads/sample. To

250 explore other cost scenarios we provide a webtool we created a webtool where one can enter

251 budget, costs, and other details about the experiment, in order to see how to achieve optimal

252 effective sample size (https://tomschwarz.shinyapps.io/RNASeqCoverageCalculator/).

\section{DISCUSSION}

254 In this work, we generate RNA-Seq data at a lower coverage than typically used in eQTL studies

255 (5.9M reads/sample) and demonstrate how this approach boosts effective sample size per unit

256 cost in an association study. To further validate this approach, we use synthetic RNA-Seq data to

257 show that the optimal level of coverage in an RNA-Seq project for the purpose of identifying eQTL

258 associations is lower than is commonly practiced ${ }^{10-12}$. Based on our findings, we recommend

259 increasing sample size while lowering sequencing depth per sample in order to achieve optimal

260 statistical power in association studies.

261 We conclude with some notes, caveats, and future directions. First, synthetic RNA-Seq via

262 downsampling reads is potentially limited in several ways. These synthetic datasets of lower

263 coverage RNA-Seq are created by uniformly sampling from real RNA-Seq data with an average

264 of 50 million reads mapped per sample. However, in practice, it is possible that sequencing biases

265 are not captured by uniform sampling due to the different experimental setup compared to the

266 dataset from which we sample ${ }^{27}$. Additionally, these synthetic datasets are based on data

267 obtained from fibroblast tissue with different transcriptomic profiles from whole blood, potentially

268 influencing the sequencing depth required to detect associations with gene expression. Finally, 
this approach is optimized for eQTL discovery. Other mechanisms that are detected using RNASeq, such as RNA splicing, have different mechanisms and will likely have different optimal

271 coverages for detection. The fact that we identify different sets of eGenes depending on which

272 gene expression measurements we consider (GTEX vs eQTLGen vs lower-coverage RNA-Seq),

273 shows that we need to increase cohort sizes in order to fully understand the connection between

274 genetics and gene expression in blood. Furthermore, the results in Figure 3A (figure showing

275 effective sample size at various coverages) indicate that even including 1490 individuals under

276 this fixed budget is not enough to achieve the optimal effective sample size. Current approaches

277 are not sufficient to understand the full landscape of eQTLs in whole blood tissue, even while only

278 considering a single genetic ancestry group. We compare the eGenes identified by GTEx,

279 eQTLGen, and the lower-coverage RNA-Seq (Supplementary Figure 8) and find that no single

280 study is sufficient in capturing all of the associations in whole blood. As observed in GWAS, much

281 larger sample sizes including far more ancestral diversity in these samples will enable discovery

282 of novel associations in transcriptomics. Including non-European populations and considering the

283 temporal aspect of gene expression will help us gain a more complete understanding of the blood

284 transcriptome landscape in the entire population.

\section{CONCLUSIONS}

286 In summary, we show that reducing coverage and increasing the number of samples in an eQTL

287 study is a valid approach for increasing effective sample size of the association study. We use

288 both real and synthetic RNA-Seq data to confirm the benefit of increased sample sizes in eQTL

289 studies. This approach can be applied to any dataset for which genotypes are available and will

290 help scientists optimize resources when measuring gene expression for the purpose of integration

291 with genetics. We provide an online tool to assist with improved design of eQTL studies at

292 https://tomschwarz.shinyapps.io/RNASeqCoverageCalculator/. 


\section{METHODS}

\section{Cohort Description}

296 The samples included are from a study with individuals ascertained for bipolar disorder (BP). The 297 cohort consists of 916 individuals with BP, 358 controls, and 216 relatives of the individuals with 298 BP.

\section{Connection between effect size and $R^{2}$}

300 If $g$ is the genotype at the SNP that we are testing for associations, and is the effect size of that 301 SNP when regressing on the true gene expression, $y$, and is the effect size of that SNP when 302 regressing on the estimated gene expression, $\tilde{y}$. The relationship between $y$ and $\hat{y}$ is as follows 303 that $r^{2}=\operatorname{corr}(y, \hat{y})$. It follows that the estimates of effect size for a SNP on the true gene 304 expression, $\hat{\beta}$, are related to the estimate of effect size for a SNP on the estimated gene 305 expression, $\widehat{\tilde{\beta}}$ as $\widehat{\widetilde{\beta}}=\operatorname{cov}(g, \tilde{y})=\operatorname{cov}(g, r y+\varepsilon)=\operatorname{cov}(g, r y)+\operatorname{cov}(g, \varepsilon)=r \widehat{\beta}$ where $\varepsilon$ is a 306 random variable with mean 0 and variance 1 . The association test statistics at low-coverage is $307 x_{\text {ground }}=N \operatorname{cor}^{2}(g, y)$ thus implying that the association statistic at low coverage

is $x_{\text {low-coverage }}=N \operatorname{cor}^{2}(g, \tilde{y})=N \widehat{\widetilde{\beta}}^{2}=N(r \widehat{\beta})^{2}=r^{2} * N \operatorname{cor}^{2}(g, y)=r^{2} x_{\text {ground }}$

\section{Budget model}

310 We modeled the cost of a large-scale bulk RNA-Seq experiment based on parameters from two

311 different library prep techniques: (1) TruSeq Stranded plus rRNA and GlobinZero and (2) TruSeq

312 Stranded polyA selected, both from the UCLA Neuroscience Genomics core. Cost, or B, is a 313 function of the following: $a$, the library preparation cost per sample, $b$, which is the target coverage 314 of each sample (in millions of reads). $c$, the cost per lane (which contains $d$ million reads), and $d$ 315 is the number of reads per sequencing lane (in millions). Altogether, we model the budget as $B=$ $316 n * a+\frac{n * b * c}{d}$

\section{Genotyping pipeline}


318 Genotypes for the low-coverage whole blood samples were obtained from the following platforms:

319 OmniExpressExome $(\mathrm{N}=810)$, PSYCH $(\mathrm{N}=523)$, and COEX $(\mathrm{N}=163)$. Given that the SNP-

320 genotype data for both the fibroblast and whole blood samples came from numerous studies using

321 various genotyping platforms (including GSA, Illumina550, OmniExpress Exome, COEX, and

322 PsychChip) the number of overlapping SNPs across all platforms was $<80 \mathrm{k}$, prompting us to

323 perform imputation separately for each genotyping platform. Genotypes were first filtered for

324 Hardy-Weinberg equilibrium $p$ value $<1.0 \mathrm{e}-6$ for controls and $p$ value $<1.0 \mathrm{e}-10$ for cases, with

325 minor allele frequency (MAF) > 0.01, leaving 148613 typed SNPs.

326 Genotypes were imputed using the 1000 Genomes Project phase 3 reference panel ${ }^{33}$ by

327 chromosome using RICOPILI v.1 ${ }^{34}$ separately per genotyping platform, then subsequently

328 merged. Imputation quality was assessed by filtering variants where genotype probability $>0.8$

329 and INFO score $>0.1$, resulting in 2289732 autosomal SNPs. We restricted to only autosomal

330 due to sex chromosome dosage, as commonly done ${ }^{12}$.

\section{Synthetic low coverage RNA-Seq}

332 We use high-coverage RNA-Seq (average of 50 million reads/sample, TruSeq Stranded plus

333 rRNA and GlobinZero library prep) from a set of 152 cell lines derived from human fibroblast cells.

334 We assume this to be the ground truth of gene expression. We used seqtk

335 (https://github.com/lh3/seqtk) to randomly sample reads at various coverages, uniformly. We

336 performed five iterations of downsampling at each level of coverage in order to account for

337 potential variability in the sampling and sequencing errors.

338 RNA-Seq processing pipeline

339 We used FASTQC to visually inspect the read quality from the lower-coverage whole blood RNA-

340 Seq (5.9M reads/sample) and the higher-coverage fibroblast RNA-Seq (13.9M reads/sample).

341 We then used kallisto to pseudoalign reads to the GRCh37 transcriptome and quantify estimates

342 for transcript expression. We aggregated transcript counts using scripts from the GTEX 343 consortium (https://github.com/broadinstitute/gtex-pipeline) ${ }^{12}$. 


\section{cis-eQTL mapping}

345 Excluding related individuals (pi_hat $>0.2$ ) from the analysis, we perform cis-eQTL analysis 346 mapping using FastQTL ${ }^{30}$, using a defined window of $1 \mathrm{Mb}$ both up and downstream of every 347 gene's TSS, for sufficiently expressed genes. We run the eQTL analysis in permutation pass 348 mode (1000 permutations, and perform multiple testing corrections using the q-value FDR 349 procedure, correcting at $5 \%$ unless otherwise specified. We then restrict our associations to the 350 top (or leading) SNP per eGene.

\section{Cell type proportion estimation}

352 We estimate the proportion of cell types of both the lower-coverage and higher-coverage bulk 353 whole blood RNA-seq datasets using CIBERSORTx ${ }^{35}$ with batch correction applied and LM22 354 signature matrix as the reference gene expression profile. The LM22 signature matrix uses 547 355 genes to distinguish between 22 human hematopoietic cell phenotypes.

$356 \quad R^{2}$ adjustment

357 To account for the variability in mapping rate across different library prep techniques ${ }^{37}$ and 358 different tissue types, we look at the mean $R^{2}$ at the expected coverage, which is calculated as 359 expected coverage $=$ target coverage * estimated mapping rate. Using mean $\mathrm{R}^{2}$ values from 360 comparing lower-coverage synthetic RNA-Seq to higher-coverage RNA-Seq real data, we fit a 361 log curve to estimate the adjusted mean $\mathrm{R}^{2}\left(\mathrm{R}_{a d j}^{2}\right)$ at the expected coverage.

\section{Effective Sample Size}

363 Under a fixed-budget setting, we calculate effective sample size $\left(N_{\text {eff }}\right)$ for a given coverage using 364 the adjusted mean $\mathrm{R}^{2}\left(R_{a d j}^{2}\right)$ and the number of samples included at a given coverage level (N) $365 \quad N_{e f f}=R_{a d j}^{2} * N$

\section{DECLARATIONS}

\section{Ethics approval and consent to participate}


368 The authors assert that all procedures contributing to this work comply with the ethical standards

369 of the relevant national and institutional committees of human experimentation and with the

370 Helsinki Declaration of 1975, as revised in 2008.

371 Consent for publication

372 Not applicable.

373

374 Availability of data and materials

375 Gene expression data will be made available upon publication

376

377 Competing interests

378 All authors declare they have no competing interest

379

380

Funding

381 T.S. was supported by the National Institute of Neurological Disorders and Stroke of the National

382 Institutes of Health under Award Number T32NS048004. T.B. was supported by the NIH (grant

383 number 5T32HG002536-19). This research was supported by the National Institute of Mental

384 Health of the National Institutes of Health under Award number 5R01MH115676-04. The content

385 is solely the responsibility of the authors and does not necessarily represent the official views of

386 the National Institutes of Health.

\section{Author Contributions}

389 T.S., B.P., and R.O. initialized the study. B.P. and R.O. directed and supervised the project. R.O.,

390 R.K., and M.P.B. collected samples. M.B. prepared samples for sequencing. Bioinformatics

391 analysis was conducted by T.S., T.B., K.H., C.D., and L.O.L. . The first draft of the manuscript

392 was drafted by T.S. and all authors contributed to editing, revisions, and approval. 


\section{Acknowledgements}

We thank the study subjects for their willingness to provide specimens and clinical data. We thank

Yi Ding, Kathryn Burch, Ruthie Johnson, Arjun Bhattacharya, and Malika Freund for meaningful discussion in helping make this work possible.

\section{SUPPLEMENTAL INFORMATION}

Supplementary Figure 1: Distribution of ancestry among samples. (S1A) MDS plot of 2000 samples in $5.9 \mathrm{M}$ read/sample cohort. (S1B) Distribution of ancestry among sample in $5.9 \mathrm{M}$ read/sample cohort. (S1C) MDS plot of 759 samples in 13.9M read/sample cohort: Genotype PC1 and PC2 are projected onto PCs from 1000 Genomes Project. (S1D) Distribution of ancestry among sample in $13.9 \mathrm{M}$ read/sample cohort.

Supplementary Figure 2: Number of pseudoaligned reads per sample. (S2A) Number of pseudoaligned reads per sample in low-coverage RNA-Seq. (S2B) Number of pseudoaligned reads per sample in high-coverage RNA-Seq.

\section{Supplementary Figure 3: Real data p-value comparison scatterplot with GTEX}

Supplementary Figure 4: Variability in correlations in synthetic data. (S4A) Scatterplot of log TPM of different draws in synthetic data. (S4B) Distribution of correlations observed between synthetic lower-coverage RNA-Seq and ground truth.

Supplementary Figure 5: Using synthetic data, how well do we capture expression as a function of average expression in a given gene. (S5A) Correlation as a function of relative expression, at 25 million reads/sample. (S5B) Correlation as a function of relative expression, at 1 million reads/sample.

Supplementary Figure 6: Using synthetic data, how well do we capture expression in different gene categories. (S6A) Using synthetic data, how well do we capture expression as a function of whether a gene is protein coding or not. (S6B) Using synthetic data, how well do we capture expression as a function of number of isoforms in a given gene. (S6C) Using synthetic RNA-Seq, how well do we capture expression as a function of gene length in a given gene Gene expression estimation accuracy simulated at 10 million reads/sample as a function of relative gene length. (S6D) Using synthetic RNA-Seq, how well do we capture expression as a function of GC content.

Supplementary Figure 7: Estimation of cell-type proportions.

Supplementary Figure 8: Overlap of significant eGenes using RNA-Seq from three different datasets

\section{REFERENCES}


439 1. Montgomery, S. B. et al. Transcriptome genetics using second generation sequencing in 440 a Caucasian population. Nature vol. 464 773-777 (2010).

$4412 . \quad$ Pickrell, J. K. et al. Understanding mechanisms underlying human gene expression

442 variation with RNA sequencing. Nature 464, 768-772 (2010).

443 3. Mancuso, N. et al. Integrating Gene Expression with Summary Association Statistics to 444 Identify Genes Associated with 30 Complex Traits. Am. J. Hum. Genet. 100, 473-487 (2017).

445 4. Gusev, A. et al. Integrative approaches for large-scale transcriptome-wide association

446 studies. Nat. Genet. 48, 245-252 (2016).

447 5. Gamazon, E. R. et al. A gene-based association method for mapping traits using

448 reference transcriptome data. Nat. Genet. 47, 1091-1098 (2015).

4496 Zhernakova, D. V. et al. Identification of context-dependent expression quantitative trait 450 loci in whole blood. Nat. Genet. 49, 139-145 (2017).

451 7. Wang, Z., Gerstein, M. \& Snyder, M. RNA-Seq: a revolutionary tool for transcriptomics.

452 Nat. Rev. Genet. 10, 57-63 (2009).

453 8. Rockman, M. V. \& Kruglyak, L. Genetics of global gene expression. Nat. Rev. Genet. 7, 454 862-872 (2006).

455 9. Gallagher, M. D. \& Chen-Plotkin, A. S. The Post-GWAS Era: From Association to 456 Function. Am. J. Hum. Genet. 102, 717-730 (2018).

457 10. Hoffman, G. E. et al. CommonMind Consortium provides transcriptomic and epigenomic 458 data for Schizophrenia and Bipolar Disorder. Sci Data 6, 180 (2019).

459 11. Franzén, O. et al. Cardiometabolic risk loci share downstream cis- and trans-gene 460 regulation across tissues and diseases. Science 353, 827-830 (2016).

461 12. GTEx Consortium. The GTEx Consortium atlas of genetic regulatory effects across 462 human tissues. Science 369, 1318-1330 (2020).

463 13. Pasaniuc, B. et al. Extremely low-coverage sequencing and imputation increases power 464 for genome-wide association studies. Nat. Genet. 44, 631-635 (2012).

465 14. Sims, D., Sudbery, I., llott, N. E., Heger, A. \& Ponting, C. P. Sequencing depth and 466 coverage: key considerations in genomic analyses. Nat. Rev. Genet. 15, 121-132 (2014).

467 15. CONVERGE consortium. Sparse whole-genome sequencing identifies two loci for major 468 depressive disorder. Nature 523, 588-591 (2015).

469 16. Homburger, J. R. et al. Low coverage whole genome sequencing enables accurate 470 assessment of common variants and calculation of genome-wide polygenic scores. Genome 471 Med. 11, 74 (2019). 
472 17. Rubinacci, S., Ribeiro, D. M., Hofmeister, R. J. \& Delaneau, O. Efficient phasing and

473 imputation of low-coverage sequencing data using large reference panels. Nat. Genet. 53, 412

474 (2021).

475 18. Baccarella, A., Williams, C. R., Parrish, J. Z. \& Kim, C. C. Empirical assessment of the

476 impact of sample number and read depth on RNA-Seq analysis workflow performance. BMC

477 Bioinformatics 19, 423 (2018).

478 19. Pritchard, J. K. \& Przeworski, M. Linkage disequilibrium in humans: models and data.

479 Am. J. Hum. Genet. 69, 1-14 (2001).

480 20. Tarazona, S., García-Alcalde, F., Dopazo, J., Ferrer, A. \& Conesa, A. Differential

481 expression in RNA-seq: a matter of depth. Genome Res. 21, 2213-2223 (2011).

482 21. Robinson, D. G. \& Storey, J. D. subSeq: determining appropriate sequencing depth

483 through efficient read subsampling. Bioinformatics 30, 3424-3426 (2014).

484 22. Krebs, C. E. et al. Whole blood transcriptome analysis in bipolar disorder reveals strong 485 lithium effect. Psychol. Med. 50, 2575-2586 (2020).

486 23. Võsa U, Claringbould A, Westra H-J, Bonder MJ, Deelen P, Zeng B, et al. Unraveling the 487 polygenic architecture of complex traits using blood eQTL meta-analysis. bioRxiv. 447367

488 (2018)

489 24. Chen, B., Khodadoust, M. S., Liu, C. L., Newman, A. M. \& Alizadeh, A. A. Profiling 490 Tumor Infiltrating Immune Cells with CIBERSORT. Methods Mol. Biol. 1711, 243-259 (2018).

$49125 . \quad$ Williams, A. G., Thomas, S., Wyman, S. K. \& Holloway, A. K. RNA-seq Data: Challenges 492 in and Recommendations for Experimental Design and Analysis. Curr. Protoc. Hum. Genet. 83, 493 11.13.1-20 (2014).

494 26. Frazee, A. C., Jaffe, A. E., Langmead, B. \& Leek, J. T. Polyester: simulating RNA-seq 495 datasets with differential transcript expression. Bioinformatics 31, 2778-2784 (2015).

496 27. Bray, N. L., Pimentel, H., Melsted, P. \& Pachter, L. Near-optimal probabilistic RNA-seq 497 quantification. Nat. Biotechnol. 34, 525-527 (2016).

498 28. Liu, Y. et al. Evaluating the impact of sequencing depth on transcriptome profiling in 499 human adipose. PLoS One 8, e66883 (2013).

500 29. Mandric, I. et al. Optimized design of single-cell RNA sequencing experiments for cell501 type-specific eQTL analysis. Nat. Commun. 11, 5504 (2020).

502 30. Ongen, H., Buil, A., Brown, A. A., Dermitzakis, E. T. \& Delaneau, O. Fast and efficient 503 QTL mapper for thousands of molecular phenotypes. Bioinformatics 32, 1479-1485 (2016). 
504 31. Marioni, J. C., Mason, C. E., Mane, S. M., Stephens, M. \& Gilad, Y. RNA-seq: an

505 assessment of technical reproducibility and comparison with gene expression arrays. Genome

506 Res. 18, 1509-1517 (2008).

507 32. Purcell, S. et al. PLINK: A Tool Set for Whole-Genome Association and Population-

508 Based Linkage Analyses. The American Journal of Human Genetics vol. 81 559-575 (2007).

509 33. Consortium, T. 1000 G. P. \& The 1000 Genomes Project Consortium. A global reference for 510 human genetic variation. Nature vol. 526 68-74 (2015).

511 34. Lam, M. et al. RICOPILI: Rapid Imputation for COnsortias PIpeLIne. Bioinformatics 36, $512930-933(2019)$.

513 35. Newman, A.M. et al. Determining cell type abundance and expression from bulk tissues 514 with digital cytometry. Nature Biotechnology 37, 773-782 (2019).

515 36. Hoffman, G. E. et al. CommonMind Consortium provides transcriptomic and epigenomic data 516 for Schizophrenia and Bipolar Disorder. Sci. Data 6, 180 (2019).

517 37. Aguet, F. et al. Genetic effects on gene expression across human tissues. Nature 550, 204$518213(2017)$

DISPLAY ITEMS

\begin{tabular}{|l|l|l|l|l|}
\hline Referred to as: & $\begin{array}{l}\text { Coverage } \\
\text { (million reads } \\
\text { per sample) }\end{array}$ & Tissue & $\begin{array}{l}\text { Number of } \\
\text { samples }\end{array}$ & Library prep method \\
\hline $\begin{array}{l}\text { Lower- } \\
\text { Coverage or M=5.9M } \\
\text { reads/sample } \\
\text { (Whole Blood) }\end{array}$ & 5.9 & $\begin{array}{l}\text { Whole } \\
\text { blood }\end{array}$ & 1490 & $\begin{array}{l}\text { TruSeq Stranded plus rRNA } \\
\text { and GlobinZero }\end{array}$ \\
\hline $\begin{array}{l}\text { Higher- } \\
\text { Coverage } \\
\text { M=13.9M } \\
\text { reads/sample } \\
\text { (Whole Blood) }{ }^{19}\end{array}$ & 13.9 & $\begin{array}{l}\text { Whole } \\
\text { blood }\end{array}$ & 570 & $\begin{array}{l}\text { Meta-analysis of (1) TruSeq } \\
\text { Stranded plus rRNA and } \\
\text { GlobinZero and (2) TruSeq } \\
\text { Stranded polyA selected }\end{array}$ \\
\hline High- & 50.3 & Fibroblast & 155 & $\begin{array}{l}\text { TruSeq Stranded polyA } \\
\text { selected }\end{array}$ \\
\hline
\end{tabular}




\begin{tabular}{|l|l|l|l|l|}
\hline $\begin{array}{l}\text { coverage } \\
\text { (Fibroblast) }\end{array}$ & 82 & $\begin{array}{l}\text { Whole } \\
\text { blood }\end{array}$ & 670 & $\begin{array}{l}\text { TruSeq Non-stranded polyA } \\
\text { selected }\end{array}$ \\
\hline GTEX $^{12}$ & N/A & $\begin{array}{l}\text { Whole } \\
\text { blood }\end{array}$ & 31684 & $\begin{array}{l}\text { Meta-analysis consisting of } \\
\text { RNA-Seq and microarray }\end{array}$ \\
\hline eQTLGen & & & & \\
\hline
\end{tabular}

Table 1: RNA-Seq datasets discussed in this paper

The coverage refers to the average number of reads that successfully map to the transcriptome, except for GTEX, which refers to the median number of total reads per sample (average mapped not available). Further description of sample overlaps among cohorts in Supplementary Note.

\begin{tabular}{|l|l|l|}
\hline & Cost per lane & Cost per sample \\
\hline Scenario 1 & $\$ 1790$ & $\$ 87$ \\
\hline Scenario 2 & $\$ 1790$ & $\$ 30$ \\
\hline Scenario 3 & $\$ 1790$ & $\$ 150$ \\
\hline Scenario 4 & $\$ 1000$ & $\$ 150$ \\
\hline
\end{tabular}

Table 2: Sequencing cost scenarios (Figure 3)

534 The cost parameters corresponding to the effective sample size scenarios in Figure 3. Cost per 535 sample reflects the cost of library prep to include an additional sample. Cost per lane reflects the cost per sequencing lane, which allows for 300 million reads. 

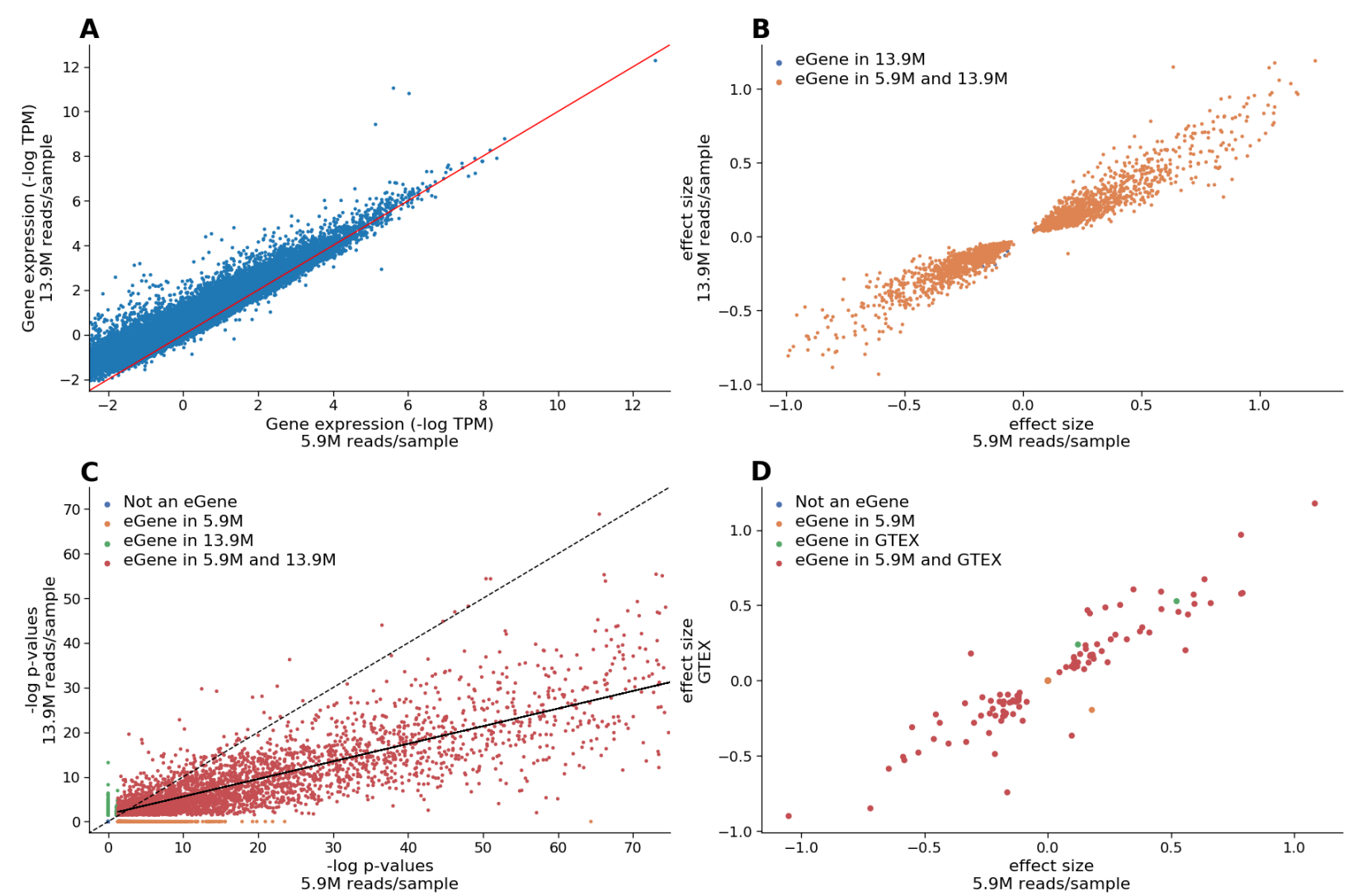

Figure 1: Concordance of eQTL discovery when using lower-coverage RNA-Seq vs highercoverage RNA-Seq (1A): Restricting to the 20735 genes with sufficient expression levels to be included in eQTL analysis in both the $5.9 \mathrm{M}$ read/sample and $13.9 \mathrm{M}$ read/sample dataset, comparison of the median expression (log TPM) across samples, of every gene. $R^{2}=0.91$. (1B): In real data, scatterplot of effect sizes of most significant eQTL hits for the 2151 protein coding genes with the same eQTL hit in both eQTL analyses performed (low-coverage and high-coverage). On the $x$-axis, we show the effect sizes for these genes using low-coverage RNA-Seq, on the $y$-axis we show the effect sizes for these genes using high-coverage RNASeq. (1C): Real data p-value comparison scatterplot: In real data, scatterplot of -log p-values of most significant eQTL hit for 13950 genes included in both eQTL analyses performed (lowcoverage and high-coverage). On the $\mathrm{x}$-axis, we show the -log $\mathrm{p}$-values for these genes using low-coverage RNA-Seq, on the $y$-axis we show the -log p-values for these genes using highcoverage RNA-Seq. The dotted line shows $y=x$, while the solid line shows the line of best fit for the 3985 protein-coding eGenes with a significant eQTL hit in both datasets. (1D): In real data, scatterplot of effect sizes of the most significant eQTL hit for the 140 eGenes with the same leading SNP identified in both eQTL analyses performed (lower-coverage RNA-Seq with 5.9M reads/sample and GTEX). On the x-axis, we show the effect size for these eGenes from eQTL analysis conducted using the 1490 individuals of EUR ancestry and imputed genotypes, and on the $y$-axis we show the effect sizes for these eGenes from eQTL analysis published by the GTEX Consortium. 

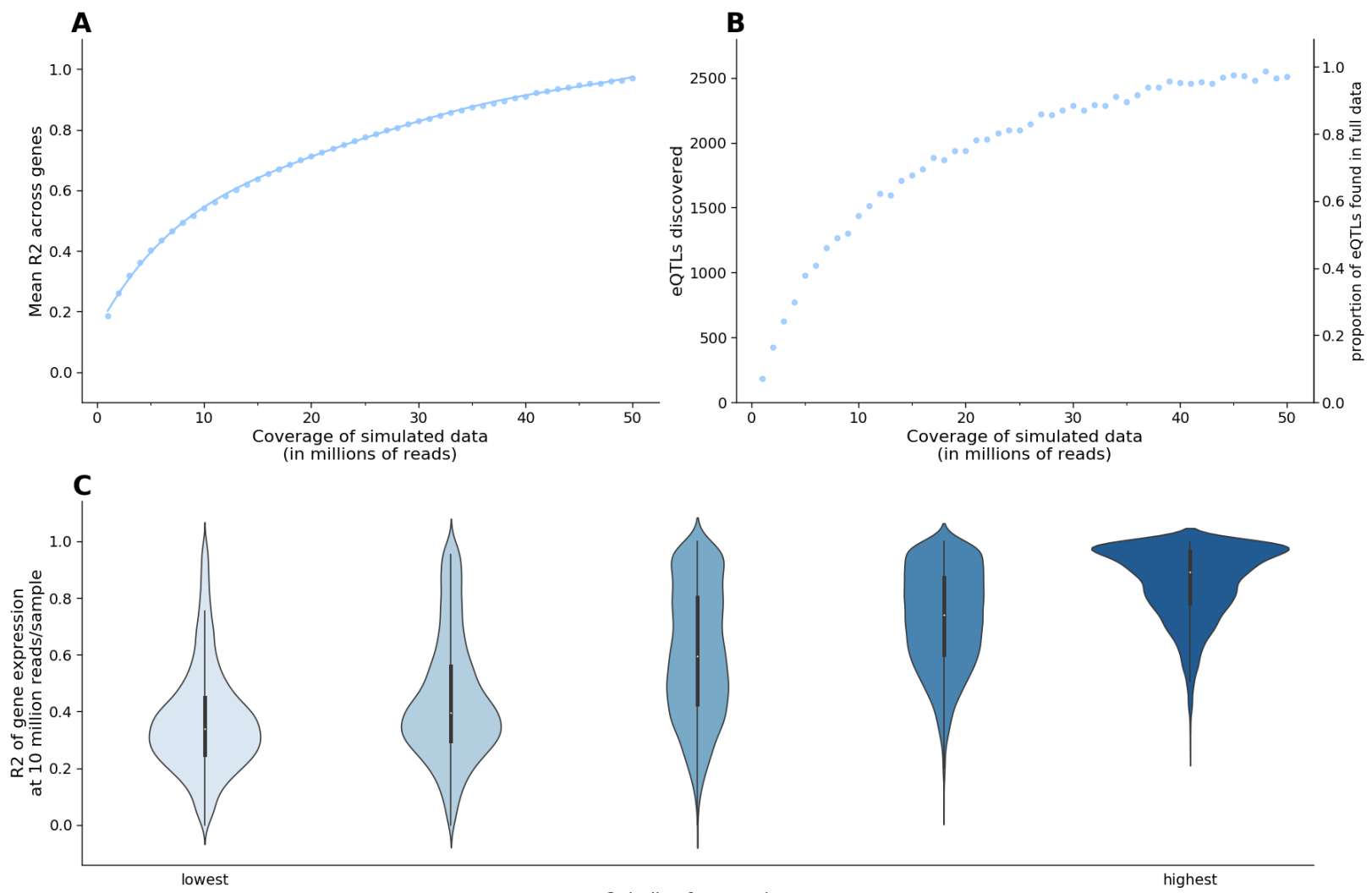

Figure 2: Synthetic lower-coverage RNA-Seq captures expression signal (2A): On the $\mathrm{x}$-axis, we show the level of simulated coverage, and on the $y$-axis we show the mean Pearson correlation of every gene. We calculate this value by finding the $R^{2}$ values for the TPM values of each of 45,910 genes across 155 samples between the high coverage data (average of 50 million reads per sample) and the simulated data, and reporting the mean $R^{2}$ value per gene. (2B): For a fixed number of individuals, absolute number and percentage of eGenes captured at $5 \%$ FDR, for synthetic RNA-Seq at varying levels of coverage. (2C): Gene expression accuracy as a function of relative gene expression observed in actual RNA-Seq data with 50 million reads/sample. 23,540 genes (with average expression $<0.1$ TPM) are divided into five ascending quintiles of expression based on their average expression in 155 samples. 

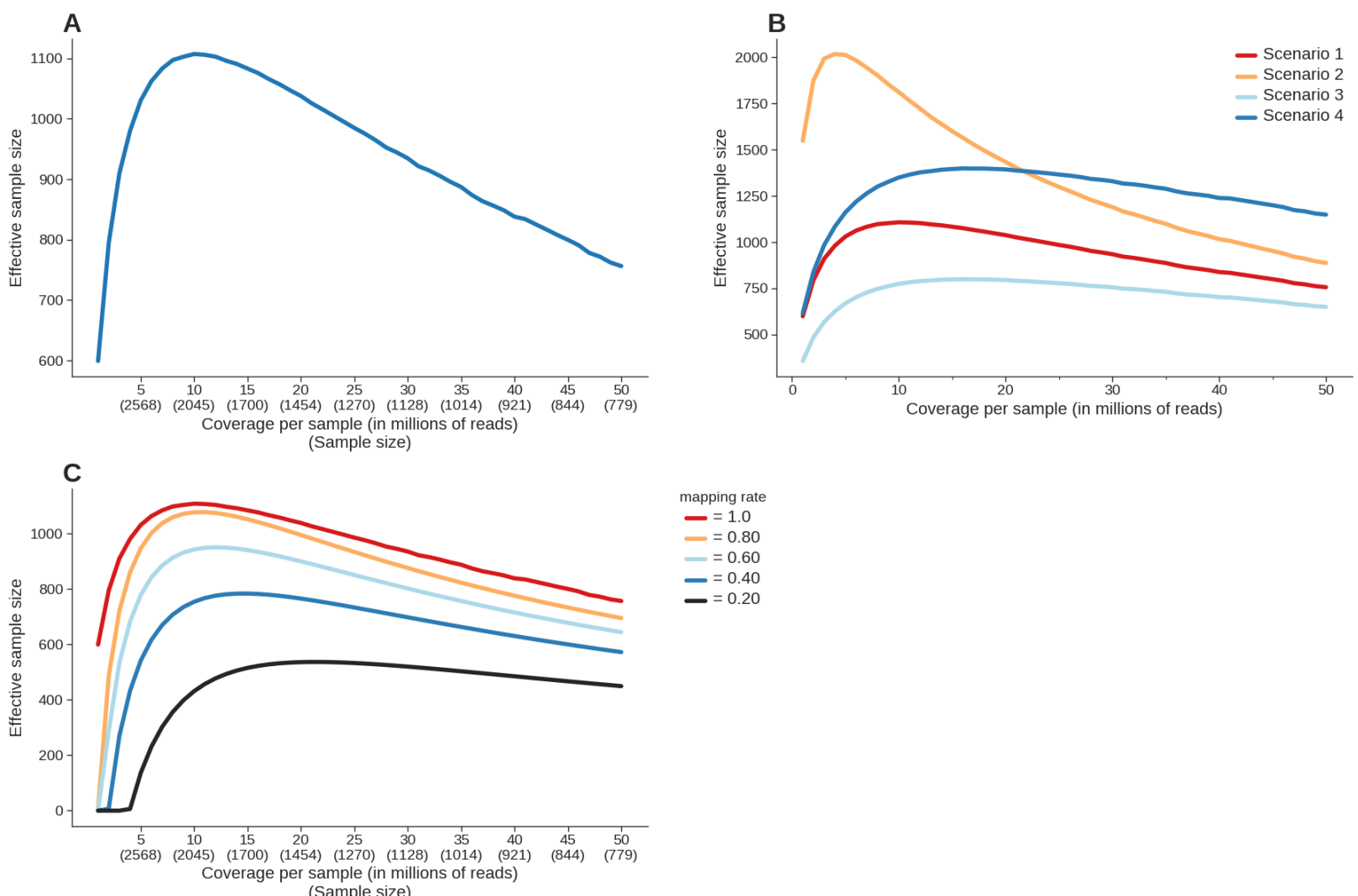

Figure 3: Effective sample size under various budget parameters. (3A): Effective sample size in RNA-Seq under a fixed budget $(\$ 300,000)$ as a function of the number of samples and the resulting coverage. Cost assumptions: $\$ 87$ per library prep per sample, $\$ 1790$ per lane of sequencing (300 million reads). (3B): Effective sample size in RNA-Seq under a fixed budget $(\$ 300,000)$ as a function of the number of samples and the resulting coverage. Cost assumptions vary and are reflected in Table 2. (3C): Effective sample size under a fixed budget $(\$ 300,000)$ as a function of the number of samples and the results coverage. A global mapping rate parameter is used to simulate actual experimental conditions (Methods). 